\title{
Dupilumab-A Potential New Biologic for Chronic Rhinosinusitis with Nasal Polyps
}

\author{
Kevin Hur and Robert C Kern
}

Department of Otolaryngology—Head and Neck Surgery, Feinberg School of Medicine, Northwestern University, Chicago, IL, USA

DOI: https://doi.org/10.17925/USPRD.2020.5.1.15

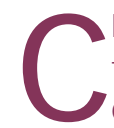
hronic rhinosinusitis with nasal polyps (CRSWNP) affects up to $2 \%$ of the general population and oftentimes confers a significant burden for patients. Dupilumab is the first monoclonal antibody approved by the United States Food and Drug Administration for the treatment of CRSWNP. Dupilumab inhibits the interleukin-4 receptor $\alpha$ (IL-4R $\alpha$ ) subunit, thereby obstructing the signaling of type 2 cytokines IL-4 and IL-13. By significantly decreasing the size of nasal polyps and improving the major symptoms associated with CRSwNP, dupilumab is an effective option, in addition to surgery and oral corticosteroids, for the treatment of CRSWNP refractory to intranasal steroids. The precise role for this drug in the management of refractory CRSWNP awaits further real-world experience.

\section{Keywords}

Dupilumab, biologic, chronic rhinosinusitis, nasal polyps, type 2 inflammation, endoscopic sinus surgery

Disclosure: Kevin Hur and Robert C Kern have nothing to disclose in relation to this article.

Review Process: Double-blind peer review.

Compliance with Ethics: This article involves a review of the literature and did not involve any studies with human or animal subjects performed by any of the authors.

Authorship: The named authors meet the International Committee of Medical Journal Editors (ICMJE) criteria for authorship of this manuscript, take responsibility for the integrity of the work as a whole, and have given final approval for the version to be published.

Access: This article is freely accessible at touchRESPIRATORY.com (c) Touch Medical Media 2020.

Received: November 27, 2020

Accepted: March 31, 2020

Published Online: April 24, 2020

Citation: US Respiratory \& Pulmonary Diseases. 2020;5(1):15-7

Corresponding Author: Robert C Kern, Department of Otolaryngology-Head and Neck Surgery, Feinberg Schoo of Medicine, Northwestern University, 675 North St Clair Street, Suite 15-200, Chicago, IL 60611, USA. E:robert.kern@nm.org

Support: No funding was received in the publication of this article.
Chronic rhinosinusitis (CRS) is a clinical syndrome defined by symptomatic inflammation of the paranasal sinuses and nasal cavity for at least 12 weeks in duration. Historically, CRS has been classified into 2 subgroups, CRS with nasal polyps (CRSWNP) and CRS without nasal polyps. Those with CRSWNP have a more significant burden of disease and poor quality of life. Current treatment options include oral corticosteroids, steroid nasal sprays, and surgery. However, patients with CRSWNP have a high rate of symptomatic polyp recurrence despite medical and surgical therapy. ${ }^{1-3} \mathrm{As}$ a consequence, additional treatment options are being developed that target specific elements of the inflammatory signaling pathways of CRSWNP. This approach most commonly utilizes monoclonal antibodies, also termed 'biologics', that are typically directed at cytokines or their receptors. These biologics act to interdict the pathway, limiting upstream progression of the inflammatory cascade.

Dupilumab (Dupixent ${ }^{\oplus}$; Sanofi, Paris, France) was the first biologic approved for the treatment for CRSWNP by the United States Food and Drug Administration (FDA) in 2019 after completion of phase II and phase III trials. ${ }^{4.5}$ The medication is self-injected biweekly by the patient, either in the thigh or abdomen. Dupilumab was initially approved in 2017 for the treatment of moderate-to-severe atopic dermatitis and in 2018 for the treatment of moderate-to-severe refractory eosinophilic or steroid-dependent asthma. ${ }^{6,7}$ The biologic is a human immunoglobulin G4 (IgG4) monoclonal antibody directed against the interleukin- 4 receptor $\alpha(I L-4 R \alpha)$ subunit. Binding of dupilumab to the IL-4R $\alpha$ subunit inhibits the signaling of both IL-4 and IL-13, two cytokines in the type 2 inflammatory pathway which is key to the pathophysiology of atopic dermatitis and asthma, as also CRSWNP.

In this review, we will discuss the pathophysiology of CRS with nasal polyps, dupilumab's mechanism of action, the clinical trials that led to dupilumab's approval by the FDA, and safety profile of this new class of therapies available for CRSWNP.

\section{Pathophysiology of chronic rhinosinusitis with nasal polyps}

About 85\% of CRSWNP in areas outside of Asia exhibit type 2 inflammation, which explains the effectiveness of corticosteroids for CRSWNP, and the potential benefit of biologics which target elements of the this pathway. ${ }^{8,9}$ The process begins with epithelial signals that stimulate type 2 innate Iymphocytes (ILC2 cells) and Th2 differentiation with the production of cytokines IL-4, IL-5, and IL13. These cytokines invoke a cascade leading to the infiltration and/or activation of large numbers of eosinophils, mast cells, and basophils. In Asian countries, patients with CRSWNP tend to have a more neutrophilic cellular predominance. Less type 2 inflammation is observed in Asian CRSWNP, who exhibit predominantly type 1 and type 3 inflammation. ${ }^{10}$ In parallel, ILC1 and ILC3 cells are activated as well as the corresponding Th1 and Th17 subsets with release of the canonical cytokines interferon- $\gamma$ and IL-17, respectively. ${ }^{11}$ Type 2 cytokines, IL-4, IL-5, and IL-13, influence several biological processes including immunoglobulin class switching to IgE and IgG4, mucus production, inflammatory cell chemotaxis with upregulation of vascular cell adhesion molecule-1, and the activation of eosinophils. ${ }^{12}$ 
The ILC cells, in general, function as the first-line defenders in the airway epithelial barrier. The epithelial signals to ILC2s are well characterized and these cells are also important sources of type 2 cytokines in CRSWNP, in addition to the Th2 lymphocytes. ${ }^{13-15}$ Importantly, dupilumab targets cytokine activity independent of the cellular source of the cytokines.

Nasal polyps are inflammatory outgrowths of the sinonasal mucosa and are associated with marked tissue edema, diminished collagen, and extracellular matrix degradation. ${ }^{16}$ The formation of polyps starts with local inflammation which leads to the leakage of fibrogen from blood vessels into the sinonasal tissue forming a matrix that traps proteins leading to tissue edema. ${ }^{17}$ The type 2 cytokine $\mathrm{IL}-13$ fosters formation of the fibrin matrix, in part via inhibition of tissue plasminogen activator, which normally would degrade the fibrin matrix. Inhibition leads to matrix stabilization, with formation and growth of nasal polyps. ${ }^{17}$ IL-13 also leads to leakiness of the epithelial barrier, which also fosters type 2 inflammation. ${ }^{8}$ It has been hypothesized that this barrier remodeling in type 2 inflammation is a key factor driving polyp recurrence. ${ }^{17}$

Asthma and CRSWNP frequently coexist and share a similar type 2 inflammatory pattern, especially in North America and Europe. As a result, several biologics targeting the Th2 inflammatory pathway in asthma, such as dupilumab, have also been investigated for the treatment of CRSWNP.

\section{Dupilumab mechanism of action}

Dupilumab binds IL-R $\alpha$ and inhibits IL-4R signaling induced by both IL-4 and IL-13, down-regulating type 2 inflammation. ${ }^{18} \mathrm{IL}-4$ and IL-13 exert their actions through three different combinations of shared receptors: one type I and two type II complexes (note that these type I and II complexes are completely unrelated to type 1, 2 and 3 inflammation terminology). ${ }^{19}$ Unfortunately, data are scant on the precise mechanism of action of dupilumab. Dupilumab has been shown to inhibit IgE production by B cells after being treated with IL-4, and inhibit IL-25-induced allergic airway inflammation and eosinophilic esophagitis in a mouse model. ${ }^{18}$ The IL-4R $\alpha$ subunit antibody could either inhibit the binding of IL-4 to the type I receptor complex, or inhibit the assembly of the type II receptor complex by preventing the recruitment of the IL-4R $\alpha$ subunit by the IL-13R $\alpha 1$ upon the binding of the latter to IL-13. The impact of an IL-4R $\alpha$ subunit antibody on the respective receptor is likely influenced by the ratio of IL-4R $\alpha$ and IL-13R $\alpha 1$ subunits in the target cells. ${ }^{19}$ If dupilumab preferentially suppressed IL-4 binding to IL-4R $\alpha$, then its effects would primarily manifest as suppressive of Th2 cell differentiation but not necessarily IL-13-driven type 2 inflammation in target tissues. The reverse would be true if dupilumab primarily suppressed IL-13 binding to the IL-4R $\alpha$ and IL-13R $\alpha 1$ subunit.

The clinical impact of which receptor complex dupilumab specifically binds to may be the observation in some patients with allergic tissue inflammation, such as asthma and eczema. Thus, if dupilumab differentially impacted the assembly of the type I receptor, the "leakage" of type II receptor signaling may explain, to some extent, the resistance of some patients to therapy. In reverse, a preference for the type II receptor may impact the capacity of dupilumab to directly act on Th2 cell-like Treg cells, which express the type I receptor, to restore their function. ${ }^{18}$ However, future studies on the interaction of dupilumab with the respective receptor complexes are needed to clarify the exact mechanism of this biologic.

\section{Therapeutic trials}

In 2019, dupilumab was approved by the FDA for the treatment of CRSWNP after a phase II trial and two phase III clinical trials. In this section, we will review the results of these studies.

A phase II, double-blind, placebo-controlled, randomized study evaluating dupilumab in 60 patients with CRSWNP was published in 2016 by Bachert et al. demonstrating reduction of nasal polyp burden at 16 weeks follow-up. Patients who had CRSwNP refractory to intranasal corticosteroids underwent either $300 \mathrm{mg}$ of dupilumab subcutaneously weekly or placebo for 16 weeks with a $600 \mathrm{mg}$ loading dose. Both groups used mometasone furoate nasal spray twice daily for the duration of the study. Eligible patients had bilateral nasal polyposis with at least extension inferior to the middle turbinate bilaterally and had symptoms of chronic sinusitis. There was a significant improvement in the dupilumab group compared to the placebo group with a least squares (LS) mean difference in the nasal polyp score by -1.6 points on a $0-8$ scale (95\% confidence interval [Cl] -2.4 to $-0.7, p<0.001$ ), Lund-Mackay CT score by -8.8 points ( $95 \% \mathrm{Cl}-11.1$ to $-6.6, p<0.001$ ), Sinonasal Outcome Test (SNOT)-22 score by -18.1 points $(95 \% \mathrm{Cl}-25.6$ to $-10.6, p<0.001)$, and the University of Pennsylvania Smell Identification Test (UPSIT) score by 14.8 points ( $95 \% \mathrm{Cl} 10.9$ to $18.7, \mathrm{p}<0.001)$. In patients with asthma, dupilumab significantly improved percentage of the forced vital capacity predicted and asthma control compared to placebo. There was also a decrease in total IgE and eotaxin-3. ${ }^{5}$ The phase II trial demonstrated significant statistical improvement in both objective and subjective outcomes in patients with CRSWNP who received dupilumab for 16 weeks. However, the clinical significance of these differences still needs to be further investigated as there is not a validated minimum clinically important difference (MCID) for most outcomes measured in the dupilumab clinical trials. Only the SNOT-22 has a validated $\operatorname{MCID}(\geq 8.5)$, which was exceeded in all trials discussed in this section. ${ }^{20}$

Two phase III studies have also been conducted, LIBERTY NP SINUS-24 and LIBERTY NP SINUS-52 (Table 1), which also assessed dupilumab as adjunctive therapy to mometasone furoate nasal spray. ${ }^{4,5}$ In SINUS-24, subjects were randomized to two arms, either dupilumab $300 \mathrm{mg}$ every 2 weeks or placebo every 2 weeks for 24 weeks. In SINUS-52, subjects were randomized to either dupilumab $300 \mathrm{mg}$ weekly for 24 weeks then every 4 weeks for 28 weeks, dupilumab 300 mg every 2 weeks for 52 weeks, or placebo every 2 weeks for 52 weeks. In both studies combined, 724 patients with severe CRSWNP (mean polyp size 5.97 , scale 0-8; nasal congestion score 2.40, scale 0-3), demonstrated significant improvement in both co-primary endpoints with dupilumab every 2 weeks. The nasal polyp score LS mean difference compared to placebo $(p<0.0001)$ were -2.06 ( $95 \% \mathrm{Cl}-2.43$ to -1.69$)$ and -1.80 ( $95 \% \mathrm{Cl}-2.10$ to -1.51$)$ in SINUS-24 and SINUS-52, respectively. The nasal congestion score LS mean difference $(p<0.0001)$ was $-0.89(95 \% \mathrm{Cl}-1.07$ to -0.71$)$ and $-0.87(95 \%$ $\mathrm{Cl}-1.03$ to -0.71$)$ in SINUS-24 and SINUS-52, respectively. Radiographic findings improved compared to placebo $(p<0.0001)$ with Lund-Mackay score LS mean difference $-7.44(95 \% \mathrm{Cl}-8.35$ to -6.53$)$ and $-5.13(95 \% \mathrm{Cl}$ -5.80 to -4.46$)$ in SINUS-24 and SINUS-52, respectively. SNOT-22 LS mean difference $(p<0.0001)$ was $-21.12(95 \% \mathrm{Cl}-25.17$ to -17.06$)$ and $-17.36(95 \%$ $\mathrm{Cl}-20.87$ to -13.85$)$ in SINUS-24 and SINUS-52, respectively. In SINUS-24, treatment effects gradually diminished in the 12-week follow-up period following drug discontinuation with worsening of the nasal polyp score 
Table 1: Summary of selected endpoints in LIBERTY NP SINUS-24 and SINUS-52 at 24 weeks

\begin{tabular}{|c|c|c|c|c|}
\hline & $\begin{array}{l}\text { LS mean } \\
\text { difference } \\
\text { versus } \\
\text { placebo }\end{array}$ & \multicolumn{2}{|l|}{$95 \% \mathrm{Cl}$} & $\mathrm{p}$ \\
\hline \multicolumn{5}{|l|}{ SINUS-24 } \\
\hline Bilateral nasal polyp score (scale 0-8) & -2.06 & -2.43 & -1.69 & $<0.01$ \\
\hline Nasal congestion score (scale 0-3) & -0.89 & -1.07 & -0.71 & $<0.01$ \\
\hline Lund-Mackay CT score (scale 0-24) & -7.44 & -8.35 & -6.53 & $<0.01$ \\
\hline Small test score (UPSIT) (scale 0-40) & 10.56 & 8.79 & 12.34 & $<0.01$ \\
\hline SNOT-22 score (scale 0-110) & -21.12 & -25.17 & -17.06 & $<0.01$ \\
\hline \multicolumn{5}{|l|}{ SINUS-52 } \\
\hline Bilateral nasal polyp score (scale 0-8) & -1.80 & -2.10 & -1.51 & $<0.01$ \\
\hline Nasal congestion score (scale 0-3) & -0.87 & -1.03 & -0.71 & $<0.01$ \\
\hline Lund-Mackay CT score (scale 0-24) & -5.13 & -5.80 & -4.46 & $<0.01$ \\
\hline Small test score (UPSIT) (scale 0-40) & 10.52 & 8.98 & 12.07 & $<0.01$ \\
\hline SNOT-22 score (scale 0-110) & -17.36 & -20.87 & -13.85 & $<0.01$ \\
\hline
\end{tabular}

$\mathrm{Cl}=$ confidence interval; $C T$ = computed tomography; $L S=$ least squares; SNOT = Sinonasal Outcome Test; UPSIT = University of Pennsylvania Smell Identification Test.

and nasal congestion. In SINUS-52, findings showed a sustained benefit throughout the treatment duration. A pooled analysis of SINUS-24 and SINUS-52 studies showed that the use of systemic corticosteroids or nasal surgery were reduced in the treatment group by $76 \%$ compared to the patients treated with $100 \mathrm{mcg}$ nasal mometasone furoate spray twice daily (34\% of placebo patients versus 10\% of dupilumab patients, $\mathrm{p}$ <0.0001). In subgroup analyses, patients with comorbid asthma, NSAID-exacerbated respiratory disease, or previous surgery had similar improvements to the overall cohort. ${ }^{4}$

\section{Safety profile}

Dupilumab is associated with side effects that should be discussed with all patients prior to treatment. In the phase II clinical trial, the most common adverse events in the dupilumab group were nasopharyngitis (47\%), injection-site reactions (40\%), and headache (20\%). ${ }^{5}$ The most frequent adverse events reported in the 24 week pooled phase III trials (SINUS-24 and SINUS-52) for the dupilumab group were nasopharyngitis (13\%), headache (7\%), epistaxis (6\%), and injection-site erythema (6\%). Interestingly, all adverse events were more commonly reported in the placebo group (74\%) versus the treatment group (69\%). ${ }^{4}$

Serious adverse events reported in the treatment arm included eosinophilia in three patients. One patient developed eosinophilic granulomatosis with polyangiitis while being treated with dupilumab, and another patient developed eosinophilia associated with arthralgia, asthma exacerbation, and insomnia during dupilumab treatment. ${ }^{4}$ Whether there is a relationship between eosinophilia and dupilumab is still unknown; however, eosinophilic granulomatosis with polyangiitis has been reported as a rare event in the administration of other biologics that target the type 2 inflammatory pathway, such as omalizumab. ${ }^{21,22}$ Whether eosinophilic granulomatosis with polyangiitis was a pre-existing condition unveiled by the cessation of systemic steroids during biologic therapy, or was secondary to the biologic itself, is unclear. Further research is necessary to clarify this possible relationship. Measuring a baseline blood eosinophil level prior to treatment and during treatment with dupilumab should be considered to monitor for this possible adverse event.

\section{Conclusion}

With consistent improvement in both objective and subjective outcomes, dupilumab is an effective nonsurgical therapeutic option in the treatment of CRSWNP not adequately controlled with nasal steroid sprays, oral corticosteroids, or surgery. The approval of dupilumab for CRSWNP patients represents a key development in the likely increasing role biologics will play in the management of patients with CRSWNP. $\square$
1. Baguley $\mathrm{C}$, Brownlow $\mathrm{A}$, Yeung $\mathrm{K}$, et al. The fate of chronic rhinosinusitis sufferers after maximal medical therapy. Int Forum Allergy Rhinol. 2014;4:525-32.

2. DeConde AS, Mace JC, Levy JM, et al. Prevalence of polyp recurrence after endoscopic sinus surgery for chronic rhinosinusitis with nasal polyposis. Laryngoscope. 2017;127:550-5.

3. Meltzer EO, Hamilos DL, Hadley JA, et al. Rhinosinusitis: establishing definitions for clinical research and patient care. J Allergy Clin Immunol. 2004;114:155-212.

4. Bachert C, Han JK, Desrosiers M, et al. Efficacy and safety of dupilumab in patients with severe chronic rhinosinusitis with nasal polyps (LIBERTY NP SINUS-24 and LIBERTY NP SINUS-52): results from two multicentre, randomised, double-blind, placebo-controlled, parallel-group phase 3 trials. Lancet. 2019;394:1638-50.

5. Bachert C, Mannent $L$, Naclerio RM, et al. Effect of subcutaneous dupilumab on nasal polyp burden in patients with chronic sinusitis and nasal polyposis: a randomized clinical trial. JAMA 2016;315:469-79.

6. Busse WW, Maspero JF, Rabe KF, et al. Liberty Asthma QUEST: phase 3 randomized, double-blind, placebo-controlled, parallel-group study to evaluate dupilumab efficacy/safety in patients with uncontrolled, moderate-to-severe asthma. Adv Ther. 2018;35:737-48.
7. Simpson EL, Bieber T, Guttman-Yassky E, et al. Two phase 3 trials of dupilumab versus placebo in atopic dermatitis. N Engl J Med. 2016;375:2335-48.

8. Stevens WW, Peters AT, Tan BK, et al. Associations between inflammatory endotypes and clinical presentations in chronic rhinosinusitis. J Allergy Clin Immunol Pract. 2019;7:2812-20.

9. Tomassen P, Vandeplas G, Van Zele T, et al. Inflammatory endotypes of chronic rhinosinusitis based on cluster analysis of biomarkers. J Allergy Clin Immunol. 2016;137:1449-56.

10. Zhang Y, Gevaert E, Lou H, et al. Chronic rhinosinusitis in Asia. J Allergy Clin Immunol. 2017;140:1230-9.

11. Bachert C, Zhang L, Gevaert P. Current and future treatment options for adult chronic rhinosinusitis: Focus on nasal polyposis. J Allergy Clin Immunol. 2015;136:1431-40.

12. Lavigne $P$, Lee SE. Immunomodulators in chronic rhinosinusitis World J Otorhinolaryngol Head Neck Surg. 2018;4:186-92.

13. Shaw JL, Fakhri S, Citardi MJ, et al. IL-33-responsive innate lymphoid cells are an important source of IL-13 in chronic rhinosinusitis with nasal polyps. Am J Respir Crit Care Med. 2013;188:432-9.

14. Mjosberg JM, Trifari S, Crellin NK, et al. Human IL-25- and IL-33-responsive type 2 innate lymphoid cells are defined by expression of CRTH2 and CD161. Nat Immunol. 2011;12:1055-62.

15. Nagarkar DR, Poposki JA, Tan BK, et al. Thymic stromal lymphopoietin activity is increased in nasal polyps of patients with chronic rhinosinusitis. J Allergy Clin Immunol. 2013;132: $593-600$

16. de Borja Callejas F, Picado C, Martinez-Anton A, et al. Differential expression of remodeling markers by tissue structure in nasal polyposis. Am J Rhinol Allergy. 2013;27:e69-74.

17. Takabayashi T, Kato A, Peters AT, et al. Excessive fibrin deposition in nasal polyps caused by fibrinolytic impairment through reduction of tissue plasminogen activator expression. Am J Respir Crit Care Med. 2013;187:49-57.

18. Harb $\mathrm{H}$, Chatila TA. Mechanisms of dupilumab. Clin Exp Allergy. 2020;50:5-14.

19. LaPorte SL, Juo ZS, Vaclavikova J, et al. Molecular and structural basis of cytokine receptor pleiotropy in the interleukin-4/13 system. Cell. 2008;132:259-72.

20. Hopkins C, Gillett S, Slack R, et al. Psychometric validity of the 22-item Sinonasal Outcome Test. Clin Otolaryngol. 2009;34: $447-54$.

21. Winchester DE, Jacob A, Murphy T. Omalizumab for asthma. N Eng/ J Med. 2006;355:1281-2.

22. Puechal $X$, Rivereau P, Vinchon F. Churg-Strauss syndrome associated with omalizumab. Eur J Intern Med. 2008;19:364-6. 